\title{
Determination of lithium in human serum by isotope dilution atomic absorption spectrometry
}

Alexander Winckelmann ${ }^{1,2}$, Dalia Morcillo ${ }^{1,2}$, Silke Richter ${ }^{2}$, Sebastian Recknagel ${ }^{2}$, Jens Riedel ${ }^{2}$, Jochen Vogl ${ }^{2}$, Ulrich Panne ${ }^{1,2}$, Carlos Abad $^{2 *}$

${ }^{1}$ Humboldt-Universität zu Berlin, Department of Chemistry, Brook-Taylor-Str. 2, 12489 Berlin, Germany

${ }^{2}$ Bundesanstalt für Materialforschung und -prüfung (BAM), Richard-Willstätter-Str. 11, 12489 Berlin, Germany

*E-mail corresponding author: carlos.abad@bam.de

\begin{abstract}
The therapeutic dose of lithium (Li) compounds, which are widely used for the treatment of psychiatric and hematologic disorders, is close to its toxic level; therefore, drug monitoring protocols are mandatory. Herein, we propose a fast, simple, and low-cost analytical procedure for the traceable determination of Li concentration in human serum, based on the monitoring of the Li isotope dilution through the partially resolved isotope shift in its electronic transition around $670.80 \mathrm{~nm}$ using a commercially available high-resolution continuum source graphite furnace atomic absorption spectrometer. With this technique, serum samples only require acidic digestion before analysis. The procedure requires three measurements - an enriched ${ }^{6} \mathrm{Li}$ spike, a mixture of a certified standard solution and spike, and a mixture of the sample and spike with a nominal ${ }^{7} \mathrm{Li} /{ }^{6} \mathrm{Li}$ ratio of 0.82 . Lanthanum has been used as an internal spectral standard for wavelength correction. The spectra are described as the linear superposition of the contributions of the respective isotopes, each consisting of a spin-orbit doublet, which can be expressed as Gaussian components with constant spectral position and width and different relative intensity, reflecting the isotope ratio in the sample. Both, the spectral constants and the correlation between isotope ratio and relative band intensity have been experimentally obtained using commercially available materials enriched with $\mathrm{Li}$ isotopes. The procedure has been validated using five human serum certified reference materials. The results are metrologically comparable and compatible to the certified values. The measurement uncertainties are comparable to those obtained by the more complex and expensive technique, isotope dilution mass spectrometry.
\end{abstract}

Keywords: lithium, human serum, isotope dilution, atomic absorption spectrometry, high-resolution continuum source graphite furnace atomic absorption spectrometry

\section{Introduction}

The therapeutic effects of lithium $(\mathrm{Li})$ on the brain and blood are well known and applied for the treatment of manic depression, granulocytopenia generated by radiation and chemotherapy, and immunoglobulin stimulation. $\mathrm{Li}$ has a narrow therapeutic range of $0.6-1.0 \mathrm{mmol} \mathrm{L}^{-1}$. Concentration of $\mathrm{Li}$ above $1.5 \mathrm{mmol} \mathrm{L}^{-1}$ in serum can be toxic [1]; hence, close monitoring of the Li concentration is mandatory to ensure effective and secure treatment. Considering the interindividual variation of the half life of $\mathrm{Li}$ in patients, a quick and accurate analysis is required [2]. Currently, the primary analytical techniques used for Li quantification are based on relative measurements, which require reference serum standards for calibration, thereby hampering traceability and comparability. 
Several analytical methods for the quantification of $\mathrm{Li}$ in biological samples have been proposed, including atomic absorption spectrophotometry (AAS) [3], inductively coupled plasma (ICP) optical emission spectroscopy (OES) [4], and ICP mass spectrometry (MS) [5]. The development of ICP-based instrumentation has facilitated the simultaneous quantification of several elements of medical interest with sufficient accuracy (trueness and precision) [6-8]. However, ICP-based methods generally require laborious sample preparation, are expensive and matrix dependent. In this context, methodologies like isotope dilution (ID) are less matrix sensitive and provide reduced uncertainties in the measurement $[9,10]$. ID is an absolute technique that improves metrological quality and provides an almost standard-free calibration approach, once the isotope spike is characterized.

Quantification of Li by ID-ICP-MS has been proposed for geological and biological samples [11,12]. ID-MS can provide analyte concentrations that are traceable to the international system of units (SI) and thus enabling comparability, when certain requirements are fulfilled [13]. However, in the case of Li, MS based methods are prone to large mass bias and matrix effects and huge instrumental drift, which is primarily due to a low mass-tocharge ratio and the resulting high relative mass difference between the two Li isotopes [14]. The high extent of the mass bias and its high fluctuation hinders its accurate correction, which in turn negatively affects the measurement uncertainty.

Some attractive alternatives to ID-MS techniques are based on the isotope shift in atomic and molecular electronic spectra. For example, ID-AAS was proposed by Brost et al. to monitor the absorption coefficient of naturally occurring $\mathrm{Li}$ in human plasma and enriched isotopic materials using natural and ${ }^{6} \mathrm{Li}$-enriched hollow cathode lamps. The bias for Li recovery in plasma ranged from $-2.8 \%$ to $0.6 \%$ [15]. However, the availability of isotopically enriched hollow cathode lamps limits the practical application of this method.

In recent years, optical spectrometry has flourished for isotope analysis. The use of a continuum light source coupled with a high-resolution echelle spectrometer and a charge-coupled device detector allows the monitoring of the electronic transition of transient diatomic molecules. The isotope shift in the electronic spectra of these diatomic molecules depends on the reduced mass and is larger than those observed in atomic spectra [16]. Thus, the monitoring of the isotope shift and the relative intensities of the isotopologue couple $\mathrm{Al}^{35} \mathrm{Cl} / \mathrm{Al}^{37} \mathrm{Cl}$ proved useful for the trace analysis of $\mathrm{Cl}$ via ID using a commercial available high-resolution continuum source graphite furnace molecular absorption spectrometer (HR-CS-GF-MAS) [17]. The same approach was applied for the trace analysis of $\mathrm{Br}$ and $\mathrm{Ca}[18,19]$. Additionally, accurate isotope ratios, which are the core of ID-MS applications, can be achieved by HR-CS-MAS and HR-CS-AAS using modern methods for data analysis like partial least square regression and machine learning, as was demonstrated for $\mathrm{B}$ and $\mathrm{Li}$, respectively [20,21]. In this latter work on $\mathrm{Li}$ isotope ratio analysis, the electronic transition $2^{2} \mathrm{P} \leftarrow 2^{2} \mathrm{~S}$ was explored. This transition can be used for $\mathrm{Li}$ quantification by ID.

This work investigates the determination of the amount of $\mathrm{Li}$ in human serum reference materials based on an ID approach applied to HR-CS-GFAAS. Since the atomic spectra of Li follow a nonlinear Gaussian function, this can be used for isotope ratio analysis for SI traceability instead of a calibration model by machine learning. The performance achieved by ID-HR-GFAAS in the present study is compared with those obtained by certificates of analysis of reference materials and previous reports on ID-MS. 


\section{Materials and Methods}

\subsection{Sample preparation}

High-purity deionized water with a resistivity of $18 \mathrm{M} \Omega \mathrm{cm}$ obtained from a Milli-Q system (Millipore gradient, Merck Millipore, Darmstadt, Germany) was used throughout the experiments. Nitric acid $\left(\mathrm{HNO}_{3}\right.$; EMSURE®, Merck, Darmstadt, Germany) was used after purification by subboiling distillation in PFA containers. The samples analyzed consisted of five serum certified reference materials (CRMs): BCR 304 (Joint Research Centre, Belgium), ERM-DA250a and ERM-DA251a (LGC Limited, UK), Seronorm L-1 (SERO AS, Norway), and Seronorm L-2 (SERO AS, Norway). A spike solution was prepared from metallic ${ }^{6} \mathrm{Li}\left(\approx 95 \%{ }^{6} \mathrm{Li}\right)$ in $2 \% \mathrm{HNO}_{3}$ with a mass concentration of around $0.5 \mathrm{mg} \mathrm{L}^{-1}$. For the ID analysis, $0.3 \mathrm{~g}$ of each serum sample was digested in

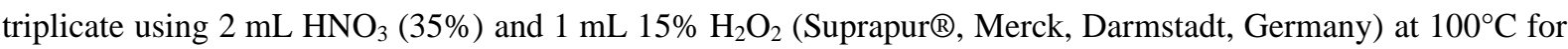
30 minutes. After digestion, $0.7 \mathrm{~g}$ of spike solution was added, and the solution was filled up to $25 \mathrm{~mL}$ with $2 \%$ $\mathrm{HNO}_{3}$. For reverse ID [9], $0.3 \mathrm{~g}$ of an ICP standard solution (traceable to NIST SRM® 3129a, Certipur®, Merck, Darmstadt, Germany) was mixed with $0.7 \mathrm{~g}$ of the spike solution and filled up to $10 \mathrm{~mL}$ with $2 \% \mathrm{HNO}_{3}$. An ${ }^{7} \mathrm{Li}^{-}$ enriched solution was used for optimization of the fit parameters and it was prepared by diluting ${ }^{7} \mathrm{Li}_{2} \mathrm{CO}_{3}(\geq 99$ atom $\%, 99 \%{ }^{7} \mathrm{Li}$, Sigma-Aldrich, USA) in $2 \% \mathrm{HNO}_{3}$ for a mass concentration of around $0.5 \mathrm{mg} \mathrm{L}^{-1}$.

\subsection{Atomic absorption spectrometry measurements}

A ContrAA 800D HR-CS-GFAAS model (Analytik Jena, Germany) with a graphite furnace (PIN platform) was used for all measurements. The wavelength of the instrument was centered at $670.7845 \mathrm{~nm}$. The optimized measurement conditions provided in our recent work were applied [21]. However, owing to the low vapor pressure of $\mathrm{La}$, the atomization temperature was increased to $2,500^{\circ} \mathrm{C}$. For each measurement, $10 \mu \mathrm{L}$ of digested sample was injected. The Li concentration of the injected solutions was adjusted to match an extinction of 3.0 integrated from 670.7361 to $670.8680 \mathrm{~nm}$. A La ICP standard solution (Certipur®, Merck, Darmstadt, Germany) was added as an internal spectral standard with a final mass concentration of $2 \mathrm{~g} \mathrm{~L}^{-1}$. Each sample was measured ten times, and 150 spectra were recorded during each atomization. The same procedure was applied for the ${ }^{6} \mathrm{Li}$ spike solution and the spiked standard solution (used for reverse ID) and for the ${ }^{7} \mathrm{Li}$ solution. Between each sample, a blank sample was measured five times.

\subsection{Data analysis}

For each measurement, 150 spectra were collected during the Li atomization and averaged. Spectral data were preprocessed and reduced using the MATLAB software (R2020a, The MathWorks Inc., USA). The spectral data of each measurement were compiled, transformed, and integrated from three-dimensional to two-dimensional spectra by converting them to their average. Using the fminsearch function in MATLAB, the spectra were fit to Equation 1, a sum of four Gaussian functions, where $c_{i}$ is the central wavelength, $w_{i}$ is the spectral width, and $A_{i}$ is the peak area.

$$
f(x)=\sum_{i=1}^{4} \frac{A_{i}}{w_{i} \sqrt{\pi / 2}} \cdot e^{-2\left(\frac{x-c_{i}}{w_{i}}\right)^{2}} \text { Equation } 1
$$

The four individual Gaussian functions correspond to two spin-orbit split isotope transitions. This results in tight constraints of the 12 variables in Equation 1, enabling a robust fitting. All four central wavelength positions 
$c_{i}$ are only governed by the term energies and can be treated as constant. The same applies for the spectral widths $w_{i}$, which are primarily determined by the instrumental resolution. Since the four peaks reflect only two doublet transitions of the two isotopes, the ratio between two respective areas (e.g., $A_{l}$ and $A_{2}$ ) is constant. Namely, the spectral displacement between $c_{1}$ and $c_{3}$ is the isotopic shift of $15.80 \mathrm{pm}$ between ${ }^{7} \mathrm{Li}$ and ${ }^{6} \mathrm{Li}$ [22]. The distance between $c_{1}$ and $c_{2}$ or $c_{3}$ and $c_{4}$ is the spin-orbit splitting of $15.08 \mathrm{pm}$. For calibration of the absolute spectral position, the La signal was fit to a single Gaussian function. The La peak center $\left(c_{L a}\right)$ was then used to correct the Li peak center. Therefore, $A_{l}$ and $A_{3}$ are the only free variables. The script used for data preprocessing and analysis are provided in the supplementary information.

\section{Results}

The ID analysis was based on the isotope shift of the electronic transition $2^{2} \mathrm{P} \leftarrow 2^{2} \mathrm{~S}$, which exhibits two spinorbit components for each isotope, as we previously described [21]. The nonlinear fit parameters were optimized using the spectra of ${ }^{7} \mathrm{Li}$ - and ${ }^{6} \mathrm{Li}$-enriched materials. The wavelength of the spectra was corrected by using La as an internal spectral standard for the electronic transition $5 \mathrm{~d} 2(3 \mathrm{~F}) 6 \mathrm{p} \leftarrow 5 \mathrm{~d} 2(3 \mathrm{~F}) 6 \mathrm{~s}$ as shown in Fig. 1 [23].

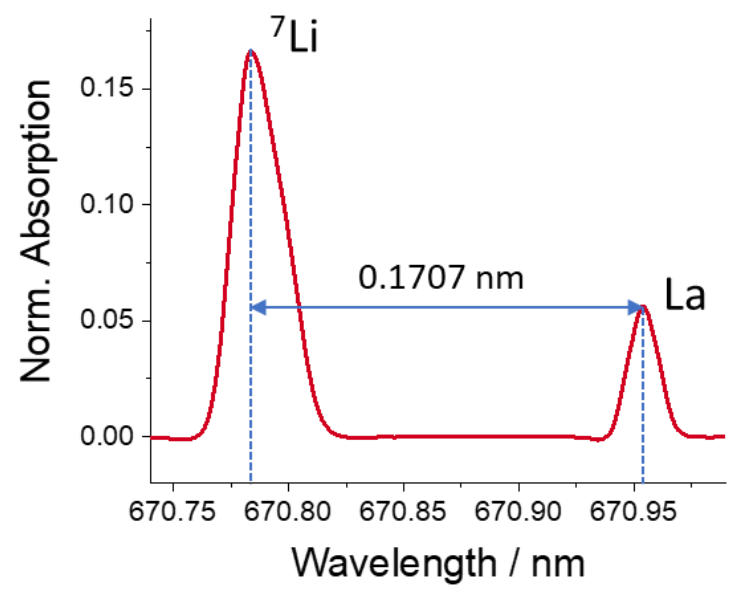

Fig. 1 Use of La as an internal spectral standard in a ${ }^{7} \mathrm{Li}$-enriched sample.

The $A_{l} / A_{2}$ ratio, which is dictated by spin statistics and the Einstein coefficients of the transitions, was determined to be 2.5645 (as well as the $A_{3} / A_{4}$ ratio) and was fixed at this value. The widths, $w_{1}$ and $w_{3}$, were determined and set to be $0.016 \mathrm{~nm}$, and the widths, $w_{2}$ and $w_{4}$, were fixed at $0.014 \mathrm{~nm}$. For data analysis without correction, the peak center $c_{l}$ was set at $670.7833 \mathrm{~nm}$. The La-corrected Li peak center was set at $c_{1}=c_{L a}-0.1707$ $\mathrm{nm}$. The ${ }^{7} \mathrm{Li} /{ }^{6} \mathrm{Li}$ isotope ratios were determined as the ratio of $A_{l} / A_{3}$. Subsequently, these ratios were fed into Equation 2 [9] to calculate the Li contents in the serum samples, where $w_{\text {sample }}$ is the Li mass fraction in the sample, $w_{\text {spike, } 6 \mathrm{Li}}$ is the ${ }^{6} \mathrm{Li}$ mass fraction in the sample, $M$ (sample) is the molar mass of $\mathrm{Li}$ in the sample, $M\left({ }^{6} \mathrm{Li}\right)$ is the molar mass of ${ }^{6} \mathrm{Li}, x_{\text {sample, }}{ }_{\mathrm{Li}}$ is the amount fraction of $\mathrm{Li}$ in the sample, $m$ is the mass of sample and added spike, and $\mathrm{R}$ represents the isotope ratio in spike, sample, and spike-sample mix, respectively. This procedure for Li quantification via ID is illustrated in Fig. 2. Natural occurring variations in the Li isotope ratios are much smaller than the uncertainties in the ID-AAS measurements. Therefore, the atomic weight interval of Li with naturally isotopic composition, i.e., between 6.9387 and $6.9438 \mathrm{~g} \mathrm{~mol}^{-1}$ was assumed in the serum samples [24]. The mass 
fraction of the spike solution was determined using reverse ID by rearranging the equation for $w_{\text {spike }}$ and setting $w_{\text {spike, }}{ }_{L i}$ as the certified concentration of the ICP standard solution.

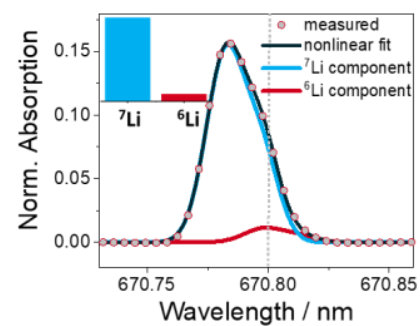

(a) Sample

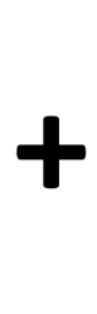

$+\quad$ (b) Spike ${ }^{6} \mathrm{Li}$

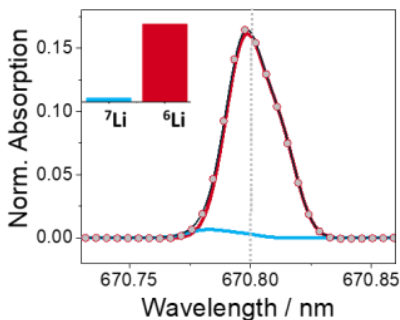

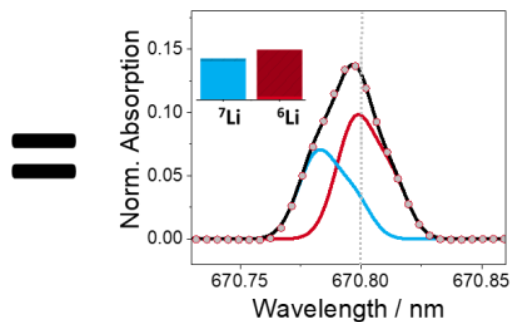

$=$ (c) Sample + Spike

Fig. 2 Representation of the isotope dilution high-resolution continuum source graphite furnace atomic absorption spectrometry-based approach for determination of Li by its isotope shift in the atomic spectra. (a) Spectrum of a sample with a naturally occurring isotopic composition ( $\mathrm{Li}$ inductively coupled plasma standard) added to a ${ }^{6} \mathrm{Li}$-enriched spike (b). The combination of (a) and (b) produced the spectrum of the mixture (c). On the top left corner of each spectrum, schematic representation of the isotope amount fractions of Li is provided.

$$
w_{\text {sample }}=w_{\text {spike },{ }^{6} L i} \frac{M(\text { sample })}{M\left({ }^{6} \mathrm{Li}\right) \cdot x_{\text {sample },{ }^{6} \mathrm{Li}}} \cdot \frac{m_{\text {spike }}}{m_{\text {sample }}} \cdot \frac{R_{\text {spike }}-R_{\text {mix }}}{R_{\text {mix }}-R_{\text {sample }}} \text { Equation } 2
$$

Table 1 summarizes the results of the Li quantification by ID-HR-CS-GFAAS. The corresponding uncertainties were calculated according to the Guide to the Expression of Uncertainty in Measurement supplement 1 (GUMS1) using GUM Workbench 2.4 (Metrodata GmbH, Germany) and Open Monte Carlo Engine v1.2.3 (Ruediger Kessel, NIST, USA). The metrological compatibility of the ID-HR-CS-GFAAS data with the certified values of the CRMs can be evaluated by applying the $E_{\mathrm{n}}$ value, which is the difference of two values divided by the expanded uncertainty of this difference [25]. Both values are considered metrologically compatible for $E_{\mathrm{n}}<1$ and not metrologically compatible for $E_{\mathrm{n}}>1$. As shown in Table 1, the Li concentrations determined by the present IDHR-CS-GFAAS method are metrologically compatible with the certified values.

Table 1 Results of isotope dilution high-resolution continuum source graphite furnace atomic absorption spectrometry (ID-HR-CS-GFAAS) and certified Li content in human serum certified reference materials (CRMs)

\begin{tabular}{|c|c|c|c|c|c|c|c|c|}
\hline \multirow[b]{2}{*}{ CRM } & \multicolumn{2}{|c|}{ Certified Li content } & \multicolumn{3}{|c|}{ ID-AAS without correction } & \multicolumn{3}{|c|}{ ID-AAS with La correction } \\
\hline & $\begin{array}{l}\text { Molarity } \\
/ \mathrm{mmol} \mathrm{L}^{-1} \mathrm{Li}\end{array}$ & $U$ & $\begin{array}{l}\text { Molarity } \\
/ \text { mmol L }{ }^{-1} \mathrm{Li}\end{array}$ & $U$ & $E_{\mathrm{n}}$ & $\begin{array}{l}\text { Molarity } \\
/ \mathrm{mmol} \mathrm{L}^{-1} \mathrm{Li}\end{array}$ & $U$ & $E_{\mathrm{n}}$ \\
\hline BCR 304 & 0.985 & 0.029 & 0.92 & 0.09 & 0.69 & 0.976 & 0.022 & 0.25 \\
\hline ERM-DA250a & $0.98 *$ & $0.06 *$ & 0.94 & 0.09 & 0.37 & 0.983 & 0.018 & 0.05 \\
\hline ERM-DA251a & $0.66^{*}$ & $0.04 *$ & 0.62 & 0.07 & 0.50 & 0.663 & 0.022 & 0.07 \\
\hline Seronorm L1 & 0.73 & 0.15 & 0.82 & 0.06 & 0.57 & 0.830 & 0.016 & 0.68 \\
\hline Seronorm L2 & 1.45 & 0.29 & 1.52 & 0.13 & 0.22 & 1.463 & 0.028 & 0.04 \\
\hline
\end{tabular}

The expanded uncertainties are expressed at the $95 \%$ confidence level $(k=2)$.

*Calculated from the certified mass fraction and its density. ERM-DA250a: $1.0294 \pm 0.0014 \mathrm{~kg} \mathrm{~L}^{-1}, \mathrm{ERM}^{-D A 251 \mathrm{a}:} 1.0175 \pm 0.0013 \mathrm{~kg} \mathrm{~L}{ }^{-1}$. Atomic weight of lithium: $6.941 \pm 0.003 \mathrm{~g} \mathrm{~mol}^{-1}[26]$. 


\section{Discussion}

A practical approach for Li quantification in human serum is presented, which is based on the partial resolution of the isotopic shift for the electronic transition $2^{2} \mathrm{P} \leftarrow 2^{2} \mathrm{~S}$ around the wavelength of $670.80 \mathrm{~nm}$ by a commercially available HR-CS-GFAAS instrument. Monitoring of this isotope shift using the La peak as a reference enables the quantification of the isotopic components with high reproducibility. Therefore, it can be used for an ID analysis by nonlinear fitting of four Gaussian curves representing the spin-orbit split doublets ${ }^{6} \mathrm{Li}_{1 / 2},{ }^{6} \mathrm{Li}{ }_{3 / 2},{ }^{7} \mathrm{Li}{ }_{1 / 2}$, and ${ }^{7} \mathrm{Li}$ 3/2, respectively. The characterization of the individual transition properties (a doublet for each isotope) can be easily performed using commercially available ${ }^{6} \mathrm{Li}$ - and ${ }^{7} \mathrm{Li}$-enriched materials. Although the HR-CS-GFAAS instrument provides high reproducibility for the wavelength measurements $( \pm 0.5$ pixels for resolution $\approx 140,000$ $\Delta \lambda / \lambda)$, the accuracy is further improved by adding La as an internal spectral standard for wavelength correction. After characterization of the Gaussian parameters, only three samples need to be measured for Li quantification: (i) a ${ }^{6} \mathrm{Li}$ spike, (ii) a mixture of the standard and ${ }^{6} \mathrm{Li}$ spike, and (iii) a mixture of the sample and ${ }^{6} \mathrm{Li}$ spike.

Additionally, the human serum samples only require acidic dilution. No matrix effects are observed, at least for the type of biological samples evaluated. The application of this procedure for Li quantification in other relevant samples having complex matrices, such as geological samples and Li-ion batteries, is under investigation. The uncertainties $(U)$ obtained using the present ID-HR-CS-GFAAS method with La correction lie between $0.016 \mathrm{mmol} \mathrm{L}^{-1}$ and $0.028 \mathrm{mmol} \mathrm{L}^{-1}$. The results are metrologically compatible with the certified reference material and comparable with those obtained with ID-AAS and ID-MS procedures, which afford uncertainties ranging between $0.006 \mathrm{mmol} \mathrm{L}^{-1}$ and $0.015 \mathrm{mmol} \mathrm{L}^{-1}[15,27,28]$. Therefore, ID-HR-CS-GFAAS can be envisaged as a metrological procedure for a fast, simple, and low-cost determination of $\mathrm{Li}$ in human serum.

\section{Acknowledgments}

The authors thank the support of Analytik Jena GmbH on this research with the HR-CS-AAS instrumentation.

\section{Conflict of interest}

There are no conflicts of interest to declare.

\section{References}

1. Young W (2009) Review of Lithium Effects on Brain and Blood. Cell Transplantation 18 (9):951-975. doi:10.3727/096368909X471251

2. Amdisen A (1990) Lithium neurotoxicity-the reliability of serum lithium measurements. Human Psychopharmacology: Clinical and Experimental 5 (3):281-285. doi:doi.org/10.1002/hup.470050324

3. Manfro ID, Tegner M, Krutzmann ME, Artmann AdC, Brandeburski MR, Peteffi GP, Linden R, Antunes MV (2020) Determination of lithium in dried blood spots and dried plasma spots by graphite furnace atomic absorption spectrometry: Method development, validation and clinical application. Talanta 216:120907. doi:10.1016/j.talanta.2020.120907 
4. Iguchi K, Usuda K, Kono K, Dote T, Nishiura H, Shimahara M, Tanaka Y (1999) Urinary Lithium: Distribution Shape, Reference Values, and Evaluation of Exposure by Inductively Coupled Plasma Argon-Emission Spectrometry. J Anal Toxicol 23 (1):17-23. doi:10.1093/jat/23.1.17

5. Goulle JP, Mahieu L, Castermant J, Neveu N, Bonneau L, Laine G, Bouige D, Lacroix C (2005) Metal and metalloid multi-elementary ICP-MS validation in whole blood, plasma, urine and hair. Reference values. Forensic Sci Int 153 (1):39-44. doi:10.1016/j.forsciint.2005.04.020

6. Laur N, Kinscherf R, Pomytkin K, Kaiser L, Knes O, Deigner H-P (2020) ICP-MS trace element analysis in serum and whole blood. PLOS ONE 15 (5):e0233357. doi:10.1371/journal.pone.0233357

7. Forrer R, Gautschi K, Lutz H (2001) Simultaneous measurement of the trace elements Al, As, B, Be, Cd, Co, $\mathrm{Cu}, \mathrm{Fe}, \mathrm{Li}, \mathrm{Mn}, \mathrm{Mo}, \mathrm{Ni}, \mathrm{Rb}, \mathrm{Se}, \mathrm{Sr}$, and $\mathrm{Zn}$ in human serum and their reference ranges by ICP-MS. Biol Trace Elem Res 80 (1):77. doi:10.1385/BTER:80:1:77

8. Bianchi F, Maffini M, Mangia A, Marengo E, Mucchino C (2007) Experimental design optimization for the ICP-AES determination of Li, Na, K, Al, Fe, Mn and Zn in human serum. J Pharm Biomed Anal 43 (2):659-665. doi:10.1016/j.jpba.2006.07.054

9. Vogl J (2007) Characterisation of reference materials by isotope dilution mass spectrometry. J Anal At Spectrom 22 (5):475-492. doi:10.1039/B614612K

10. Brun V, Masselon C, Garin J, Dupuis A (2009) Isotope dilution strategies for absolute quantitative proteomics. Journal of Proteomics 72 (5):740-749. doi:10.1016/j.jprot.2009.03.007

11. Kramer U, Kress M, Reinauer H, Spannagl M, Kaiser P (2013) Candidate reference measurement procedures for chloride, potassium, sodium, calcium, magnesium, and lithium by inductively coupled plasma (isotope dilution) sector field mass spectrometry (ICP-(ID) SFMS) in serum. Clin Lab 59 (9-10):1017-1029. doi:10.7754/clin.lab.2012.120902

12. Moriguti T, Makishima A, Nakamura E (2004) Determination of Lithium Contents in Silicates by Isotope Dilution ICP-MS and its Evaluation by Isotope Dilution Thermal Ionisation Mass Spectrometry. Geostand Geoanal Res 28 (3):371-382. doi:10.1111/j.1751-908X.2004.tb00756.x

13. Milton MJT, Wielgosz RI (2000) Uncertainty in SI-traceable measurements of amount of substance by isotope dilution mass spectrometry. Metrologia 37 (3):199. doi:10.1088/0026-1394/37/3/3

14. Lin J, Liu Y, Hu Z, Yang L, Chen K, Chen H, Zong K, Gao S (2016) Accurate determination of lithium isotope ratios by MC-ICP-MS without strict matrix-matching by using a novel washing method. J Anal At Spectrom 31 (2):390-397. doi:10.1039/c5ja00231a

15. Brost DF, Brackett JM, Busch KW (1979) Determination of lithium by optically monitored stable isotope dilution. Anal Chem 51 (9):1512-1516. doi:10.1021/ac50045a035

16. Russo RE, Bol'shakov AA, Mao X, McKay CP, Perry DL, Sorkhabi O (2011) Laser Ablation Molecular Isotopic Spectrometry. Spectrochim Acta, Part B 66 (2):99-104. doi:10.1016/j.sab.2011.01.007 
17. Nakadi FV, da Veiga MAMS, Aramendía M, García-Ruiz E, Resano M (2015) Chlorine isotope determination via the monitoring of the $\mathrm{AlCl}$ molecule by high-resolution continuum source graphite furnace molecular absorption spectrometry - a case study. Journal of Analytical Atomic Spectrometry 30 (7):1531-1540. doi:10.1039/c5ja00055f

18. Nakadi FV, da Veiga MAMS, Aramendia M, Garcia-Ruiz E, Resano M (2016) Br isotope determination via the monitoring of $\mathrm{CaBr}$ transitions using high-resolution continuum source graphite furnace molecular absorption spectrometry. Potential for direct determination of $\mathrm{Br}$ in solid samples using isotope dilution. J Anal At Spectrom 31 (7):1381-1390. doi:10.1039/C6JA00114A

19. Zanatta MBT, Nakadi FV, da Veiga MAMS (2018) CaI and SrI molecules for iodine determination by highresolution continuum source graphite furnace molecular absorption spectrometry: Greener molecules for practical application. Talanta 179:563-568. doi:10.1016/j.talanta.2017.11.052

20. Abad C, Florek S, Becker-Ross H, Huang M-D, Heinrich H-J, Recknagel S, Vogl J, Jakubowski N, Panne U (2017) Determination of boron isotope ratios by high-resolution continuum source molecular absorption spectrometry using graphite furnace vaporizers. Spectrochim Acta, Part B 136 (Supplement C):116-122. doi:10.1016/j.sab.2017.08.012

21. Winckelmann A, Nowak S, Richter S, Recknagel S, Riedel J, Vogl J, Panne U, Abad C (2021) High-Resolution Atomic Absorption Spectrometry Combined with Machine Learning Data Processing for Isotope Amount Ratio Analysis of Lithium. Preprint ChemRxiv. doi:10.26434/chemrxiv.13583024.v1

22. Moore CE (1971) Atomic Energy Levels as Derived from the Analysis of Optical Spectra. In: Nat. Stand. Ref. Data Ser., NSRDS-NBS 35 Vol. I (Reprint of NBS Circ. 467, Vol. I, 1949), 359 pp. (Nat. Bur. Stand., U.S.). doi:10.6028/NBS.NSRDS.35v1

23. Hartog EAD, Palmer AJ, Lawler JE (2015) Radiative lifetimes and transition probabilities of neutral lanthanum. Journal of Physics B: Atomic, Molecular and Optical Physics 48 (15). doi:10.1088/0953$4075 / 48 / 15 / 155001$

24. Laeter JRd, Böhlke JK, Bièvre PD, Hidaka H, Peiser HS, Rosman KJR, Taylor PDP (2003) Atomic weights of the elements. Review 2000 (IUPAC Technical Report). Pure Appl Chem 75 (6):683-800. doi:10.1351/pac200375060683

25. Vogl J, Rosner M, Kasemann SA, Kraft R, Meixner A, Noordmann J, Rabb S, Rienitz O, Schuessler JA, Tatzel M, Vocke RD (2020) Intercalibration of Mg Isotope Delta Scales and Realisation of SI Traceability for Mg Isotope Amount Ratios and Isotope Delta Values. Geostand Geoanal Res 44 (3):439-457. doi:10.1111/ggr.12327

26. Meija J, Coplen TB, Berglund M, Brand WA, De Bièvre P, Gröning M, Holden NE, Irrgeher J, Loss RD, Walczyk T, Prohaska T (2016) Atomic weights of the elements 2013 (IUPAC Technical Report). Pure Appl Chem 88 (3):265-291. doi:10.1515/pac-2015-0305

27. Michiels E, De Bievre P (1983) Accurate assay of lithium by isotope dilution mass spectrometry. International Journal of Mass Spectrometry and Ion Physics 48:369-372. doi:10.1016/0020-7381(83)87104-6 
28. Lamberty A, De Bièvre P (1993) CBNM - determination of Li in BCR reference materials RM 303 and RM 304 lyophilized serum by isotope dilution mass spectrometry (IDMS). Fresenius' Journal of Analytical Chemistry 345 (2):330-334. doi:10.1007/BF00322624 